\title{
COMBINED ACCRETION DISK AND NONTHERMAL SOURCE MODEL
} FOR AGN

\author{
D. L. Band \\ U.C. Berkeley and Lawrence Livermore National Laboratory \\ L-22, P. O. Box 808, Livermore, CA 94550 \\ M. A. Malkan \\ Astronomy Department, UCLA \\ Los Angeles, CA 90024
}

We model the infrared through gamma ray AGN spectrum with a combined accretion disk and nonthermal source model and fit the spectra of a number of sources. The accretion disk model of Sun and Malkan (Sun 1987) adds relativistic effects to the emission from an optically thick, geometrically thin accretion disk; further details can be found in Sun and Malkan (1988) in these proceedings. In the nonthermal source model of Band and Grindlay (1986) relativistic electrons radiate the infrared continuum by synchrotron emission, and the X-ray spectrum by inverse Compton scattering of ultraviolet photons from the accretion disk. The electron distribution consists of a flat $\left(n \propto \gamma^{\sim-2.4}\right)$ low-energy component, and a steeper $\left(n \propto \gamma^{\sim-3.4}\right)$ high-energy component.

So far we have modeled 10 AGN for which the necessary observables have been determined. The nonthermal model is particularly sensitive to the far-infrared turnover frequency $\nu_{T}$ which has been detected in several objects. In some objects with an apparent turnover, $\nu_{T}$ may be poorly determined due to thermal emission external to the nucleus and additional spectral features predicted by the nonthermal model. For those objects with a soft X-ray excess we assume that the disk is inclined since relativistic effects increase the high frequency flux from an inclined disk; otherwise we assume that the disk is face-on. For a given observed optical flux an inclined disk has a larger mass.

We find that the typical AGN in our sample has a mass of $M_{b h} \sim 10^{8} M_{\odot}$, a total thermal and nonthermal luminosity $\sim 0.5 L_{\text {edd }}$, and an accretion rate $\dot{m} \sim 1 M_{\odot}-\mathrm{yr}^{-1}$. The high accretion rate indicates that the thin accretion disk approximation is only marginally acceptable. Since the AGN lifetime is short, $t_{l}=M_{b h} / \dot{m} \sim 10^{8} \mathrm{yr}$, the AGN phenomenon in a given source lasts a short time, which favors Density Evolution. Further implications of the accretion disk model are discussed by Sun (1987) and Sun and Malkan (1988).

The nonthermal source has a radius of $R \sim 2 \times 10^{15} \mathrm{~cm}$, a relativistic electron density of $n_{e} \sim 5 \times 10^{6} \mathrm{~cm}^{-3}$, and a magnetic field $B \sim 200$ gauss. Consequently, the photon and magnetic fields are in rough equipartition, while the relativistic electron energy density is smaller by a factor of $\sim 10^{3}$. In an inhomogeneous source the electron density could be greater. The luminosities of the nonthermal infrared, ultraviolet accretion disk, and nonthermal X-ray to gamma ray continua are comparable (as are therefore the photon energy densities in each component). From these empirical results we can deduce the physical conditions in the nonthermal source. 
As has been found previously, the nonthermal electron lifetime is much shorter than crossing time. Therefore, electrons must be continuously injected throughout the source. To avoid a large density of thermalized electrons, the relativistic electrons must either be destroyed (e.g., in an $e^{+}-e^{-}$plasma) or recycled by reacceleration. The two component nonthermal electron distribution necessary for this model can be explained by continuous electron injection with radiative losses dominating at high energies, and an energyindependent loss mechanism at low energies. For example, electrons with an energy below the break energy may be absorbed into a reacceleration center (an energy independent loss) before they radiate away their energy; the break energy would then reflect the distribution of reacceleration centers.

The importance of photon-photon pair creation is measured by the compactness $l_{\gamma \gamma}=$ $\left(L_{e} / R\right)\left(\sigma_{T} / 4 \pi m c^{3}\right)$ (note the factor of $4 \pi$ in the definition), where $L_{e}$ is the luminosity around $\nu_{m}=m c^{2} / h$. Extrapolating the observed X-ray flux within the context of our model we find that $l_{\gamma \gamma} \sim 0.6$ when $L_{e}=\left[\nu L_{\nu}\right]\left(\nu_{m}\right)$. With $l_{\gamma \gamma}$ in this range pair creation reduces the gamma ray spectrum, but the resulting pairs do not contribute significantly to the relativistic electron distribution (Band 1987).

Since it is theoretically easier to accelerate protons than electrons, many models have been proposed wherein relativistic electrons are secondaries produced by proton-proton collisions. The energy injected in accelerating protons is radiated only when these relativistic protons interact with thermal protons. However, the relativistic proton energy density $u_{r p}$ must exceed the resulting nonthermal photon energy density $u_{n t}$ by a factor of $u_{r p} / u_{n t} \sim 150 \tau_{p}$, where we have assumed an energy conversion efficiency of $f \sim 0.3$ and the proton-proton $\sigma \sim \frac{1}{30} \sigma_{T} ; \tau_{p}$ is the scattering optical depth through the nonthermal source due to all electrons. But $\tau_{p}>1$ would be apparent in the downscatter of high energy $\mathrm{X}$-rays, the absorption of low energy $\mathrm{X}$-rays, and an increase in the variability timescale. Thus $u_{r p}$ would dominate all other energy densities. If the relativistic protons are not confined to the source then only a small fraction of the energy in relativistic protons is converted into nonthermal radiation; the extent of the nonthermal source would require explanation. On the other hand, it is not clear how the relativistic protons can be confined given their large energy density. In particular, we find that confinement by an accretion shock is unlikely (Band and Malkan 1988).

Assuming the gamma ray break in the spectrum (observed in only 3-4 sources) is at $\nu \sim m c^{2} / h$, the spectrum's Compton temperature is $T_{c} \sim 5 \times 10^{6} \mathrm{~K}$. This low $T_{c}$ rules out two co-existing phases of the BLR, which is illuminated by this spectrum. This spectrum is inconsistent with pressure confinement of the BLR clouds.

This work was supported in part by a grant from IGPP-LLNL, by NSF grant AST86-14510 (M.A.M.), and by the U.S. Department of Energy, under contract No. W-7405ENG-48 to the Lawrence Livermore National Laboratory (D.L.B.).

Band, D. L. 1987, Ap. J., 321, 80.

Band, D. L., and Grindlay, J. E. 1986, Ap. J., 311, 595.

Band, D. L., and Malkan, M. A. 1988, in preparation.

Sun, W. H. 1987, thesis, UCLA.

Sun, W. H. and Malkan, M. A. 1988, these proceedings. 